\title{
The Odd Log-Logistic Burr-X Family of Distributions: Properties and Applications
}

\author{
Hamid Karamikabir ${ }^{1}$, Mahmoud Afshari ${ }^{1, *}$, Morad Alizadeh $^{1}$, Haitham M. Yousof ${ }^{2}$ \\ ${ }^{1}$ Department of Statistics, Faculty of Intelligent Systems Engineering and Data Science, Persian Gulf University, Bushehr, 7516913798, Iran. \\ ${ }^{2}$ Department of Statistics, Mathematics and Insurance, Benha University, Benha, Egypt
}

\section{ARTICLE INFO}

\section{Article History}

Received 01 Sep 2020

Accepted 15 Jun 2021

\section{Keywords}

Odd log-logistic-G family Burr-X family

Maximum likelihood method Least square method

Weighted least square method Moments

2010 Mathematics Subject Classification: 60E05, 62F10, 62G05

\section{ABSTRACT}

In this paper, a new class of distributions called the odd log-logistic Burr-X family with two extra positive parameters is introduced and studied. The new generator extends the odd log-logistic and Burr X distributions among several other well-known distributions. We provide some mathematical properties of the new family including asymptotics, moments, moment-generating function and incomplete moments. Different methods have been used to estimate its parameters such as maximum likelihood, least squares, weighted least squares, Cramer-von-Mises, Anderson-Darling and right-tailed Anderson-Darling methods. We evaluate the performance of the maximum likelihood estimators in terms of biases and mean squared errors by means of a simulation study. Finally, the usefulness of the family is illustrated by means of three real data sets. The new models provide consistently better fits than other competitive models for these data sets. The new family is suitable for fitting different real data sets, the odd log-logistic Burr-X Normal model is used for modeling bimodal and skewed data sets and can be sued as an alternative to the gamma-normal, beta-normal, McDonald-normal, Marshall-Olkin-normal, Kumaraswamy-normal, Zografos-Balakrishnan and Log-normal distributions.

(C) 2021 The Authors. Published by Atlantis Press B.V.

This is an open access article distributed under the CC BY-NC 4.0 license (http://creativecommons.org/licenses/by-nc/4.0/).

\section{INTRODUCTION}

The odd log-logistic (OLL) family of distributions was originally developed by Gleaton and Lynch [1] they called this family the generalized log-logistic (GLL) family. Recently, many OLL-G families have been developed and studied such as the generalized OLL family by Cordeiro et al. [2] and a new generalized OLL family of distributions by Haghbin et al. [3]. The cumulative distribution function (cdf) of this family is given by the following:

$$
F_{O L L-G}(x)=F(x, \alpha, \xi)=\frac{G(x, \xi)^{\alpha}}{G(x, \xi)^{\alpha}+\bar{G}(x, \xi)^{\alpha}},
$$

Yousof et al. [4] introduced Burr X family of distributions. The cdf of this family is given by the following:

$$
F_{B u X-G}(x)=F(x ; \theta, \xi)=\left\{1-\exp \left[-\left(\frac{G(x ; \xi)}{\bar{G}(x \xi)}\right)^{2}\right]\right\}^{\theta} .
$$

Based on T-X idea (Alzaatreh et al. [5]), a new extended class called the The OLL Burr-X family of distributions (OLLBuX) is defined with cdf

$$
F(x)=\frac{\left\{1-\exp \left[-\left(\frac{G(x)}{\bar{G}(x)}\right)^{2}\right]\right\}^{\alpha \theta}}{\left\{1-\exp \left[-\left(\frac{G(x)}{\bar{G}(x)}\right)^{2}\right]\right\}^{\alpha \theta}+\left(1-\left\{1-\exp \left[-\left(\frac{G(x)}{\bar{G}(x)}\right)^{2}\right]\right\}^{\theta}\right)^{\alpha}}
$$


and probability density function (pdf)

$$
f(x)=\frac{2 \alpha \theta g(x) G(x) e^{-\left(\frac{G(x)}{\bar{G}(x)}\right)^{2}}\left\{1-\exp \left[-\left(\frac{G(x)}{\bar{G}(x)}\right)^{2}\right]\right\}^{\alpha \theta-1}\left(1-\left\{1-\exp \left[-\left(\frac{G(x)}{\bar{G}(x)}\right)^{2}\right]\right\}^{\theta}\right)^{\alpha-1}}{\bar{G}(x)^{3}\left(\left\{1-\exp \left[-\left(\frac{G(x)}{\bar{G}(x)}\right)^{2}\right]\right\}^{\alpha \theta}+\left\{1-\left\{1-\exp \left[-\left(\frac{G(x)}{\bar{G}(x)}\right)^{2}\right]\right\}^{\theta}\right\}^{\alpha}\right)^{2}} .
$$

The hazard rate function (hrf) $h(x)=f(x) /[1-F(x)]$ given by the following:

$$
h(x)=\frac{2 \alpha \theta g(x) G(x) e^{-\left(\frac{G(x)}{\bar{G}(x)}\right)^{2}}\left\{1-\exp \left[-\left(\frac{G(x)}{\bar{G}(x)}\right)^{2}\right]\right\}^{\alpha \theta-1}\left\{1-\left\{1-\exp \left[-\left(\frac{G(x)}{\bar{G}(x)}\right)^{2}\right]\right\}^{\theta}\right\}^{-1}}{\bar{G}(x)^{3}\left\{\left\{1-\exp \left[-\left(\frac{G(x)}{\bar{G}(x)}\right)^{2}\right]\right\}^{\alpha \theta}+\left\{1-\left\{1-\exp \left[-\left(\frac{G(x)}{\bar{G}(x)}\right)^{2}\right]\right\}^{\theta}\right\}^{\alpha}\right\}} .
$$

If $U \sim U(0,1)$, then we can write as follows:

$$
X_{U}=Q_{G}\left(\frac{\left\{-\log \left[1-\frac{\frac{1}{U \frac{\alpha}{\alpha \theta}}}{\left[U^{\frac{1}{\alpha}}+(1-U)^{\frac{1}{\alpha}}\right]^{\frac{1}{\theta}}}\right]\right\}^{\frac{1}{2}}}{1+\left\{-\log \left[1-\frac{u^{\frac{1}{\alpha \theta}}}{\left[\frac{1}{U^{\alpha}+(1-U)^{\frac{1}{\alpha}}}\right]^{\frac{1}{\theta}}}\right]\right\}^{\frac{1}{2}}} .\right.
$$

An interpretation of the OLLBuX-G family can be given as follows. Let $T$ be a random variable describing a stochastic system by the cdf $F_{B u X-G}(x)$. If the random variable $X$ represents the odds, the risk that the system following the lifetime $T$ will be not working at time $x$ is given by $F_{B u X-G}(x) /\left[1-F_{B u X-G}(x)\right]$. If we are interested in modeling the randomness of the odds by the log-logistic cdf $R(t)=\frac{t^{\alpha}}{1+t^{\alpha}}($ for $t$ $>0)$, the cdf of $X$ is given by the following:

$$
\operatorname{Pr}(X \leq x)=R\left[\frac{F_{B u X-G}(x)}{1-F_{B u X-G}(x)}\right]=\frac{\left\{1-\exp \left[-\left(\frac{G(x)}{\bar{G}(x)}\right)^{2}\right]\right\}^{\alpha \theta}}{\left\{1-\exp \left[-\left(\frac{G(x)}{\bar{G}(x)}\right)^{2}\right]\right\}^{\alpha \theta}+\left(1-\left\{1-\exp \left[-\left(\frac{G(x)}{\bar{G}(x)}\right)^{2}\right]\right\}^{\theta}\right)^{\alpha}} .
$$

The basic justifications for generating a new distribution in practice are the following:

1 - to make the kurtosis more fexible compared to that of the baseline distribution;

2 - to provide consistently better fits than other generated distributions with the same underlying model;

3 - to dens special models with all types of hrf;

4 - to generate distributions with left-skewed, right-skewed, symmetric, or reversed-J shape;

5 - to produce a skewness for symmetrical models;

6 - to construct heavy-tailed distributions for modelling various real data sets.

In this paper, the additional parameter $\theta$ is pursued as a tool to furnish a more flexible family of distributions which is clearly demonstrated in Section 2.

In fact, many other families can be cited such as the beta generalized-G family by Eugene et al. [6], a new method for generating families of continuous distributions by Alzaatreh et al. [5], Odd-Burr generalized family by Aizadeh et al. [7], Kumaraswamy transmuted-G by Afify 
et al. [8], univariate continuous exponential power series distributions by Ahsanullah et al. [9], exponentiated generalized-G Poisson by Aryal and Yousof [10], Topp-Leone OLL family by de Brito et al. [11], Type I general exponential class of distributions by Hamedani et al. [12], extended generalized Gompertz distribution by Karamikabir et al. $[13,14]$ among others.

The rest of the paper is outlined as follows. Two special models of the new family are presented in Section 2. We provide a linear representation for the new family in Section 3. In Section 4, we derive some mathematical properties of this family. Maximum likelihood estimation for the model parameters under uncensored data is addressed in Section 5. Simulation studies to assess the performance of the estimators are provided in Section 6. In Section 7, the potentiality of the proposed models is illustrated by means of two real data sets. In Section 8 , we offer some concluding remarks.

\section{SPECIAL CASE}

In this section we consider two special cases of propose family.

\subsection{The OLLBuX-Normal Family (OLLBuX-N)}

By taking $G(x ; \xi)$ and $g(x ; \xi)$ in (2) to be the cdf and pdf of the normal $N\left(\mu, \sigma^{2}\right)$ distribution, respectively, where $\xi=(\mu, \sigma)^{T}$, and $w(x)=$ $\exp \left(-\left[\frac{\phi\left(\frac{x-\mu}{\sigma}\right)}{1-\phi\left(\frac{x-\mu}{\sigma}\right)}\right]^{2}\right),(x \in R)$, then the OLLBuX-N density function is given by the following:

$$
f(x ; \alpha, \theta, \mu, \sigma)=\frac{2 \alpha \theta \phi\left(\frac{x-\mu}{\sigma}\right) \phi\left(\frac{x-\mu}{\sigma}\right) w(x)\{1-w(x)\}^{\alpha \theta-1}\left\{1-\{1-w(x)\}^{\theta}\right\}^{\alpha-1}}{\left[1-\phi\left(\frac{x-\mu}{\sigma}\right)\right]^{-3}\left\{\{1-w(x)\}^{\alpha \theta}+\left\{1-\{1-w(x)\}^{\theta}\right\}^{\alpha}\right\}^{2}},
$$

where $\phi\left(\frac{x-\mu}{\sigma}\right)$ and $\phi\left(\frac{x-\mu}{\sigma}\right)$ are the pdf and cdf of $N(\mu, \sigma)$, respectively. If $X$ be a random variable with density function (4), then it will be displayed by $O L L B u X-N(\alpha, \theta, \mu, \sigma)$. In Figure 1 some pdfs for OLLBuX-N have been drown.

One can see in the curves of Figure 1 that different skewed density functions including mild and high skewed ones (positive and negative) have been produced. The OLLBuX-N family build the very flexible density function (unimodal and bimodal) that is useful in fitting real data sets.
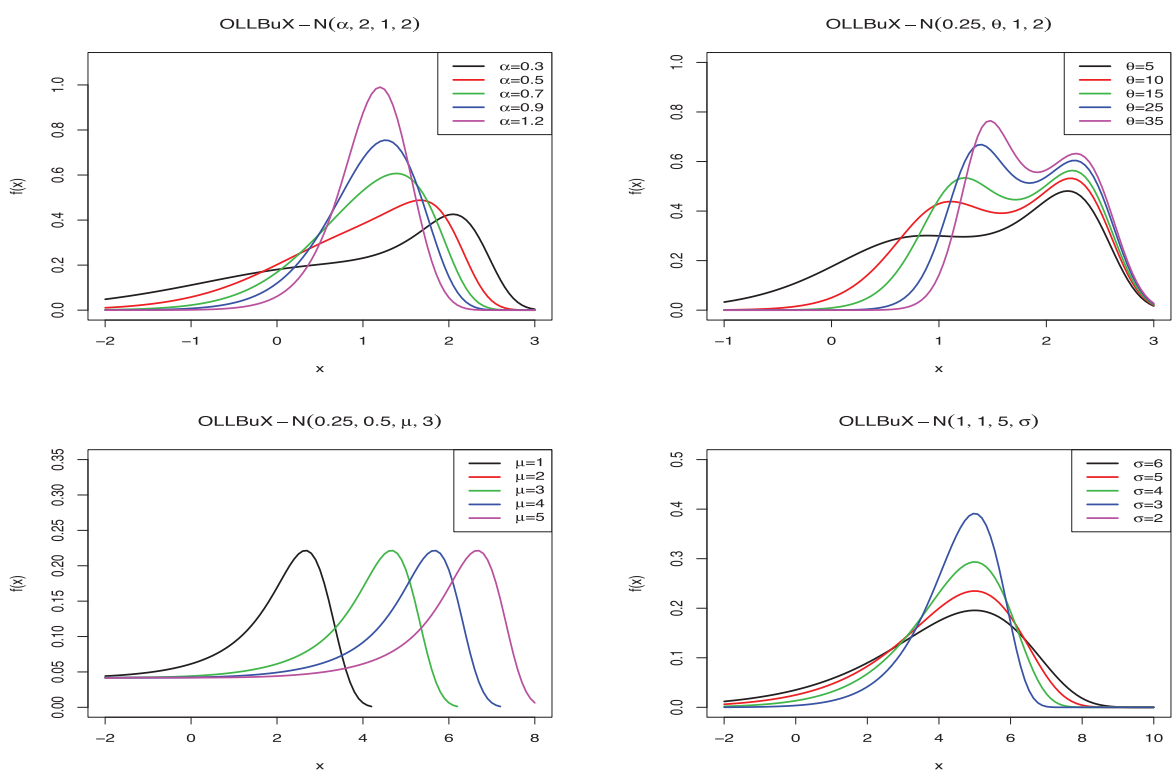

Figure 1 The sample curves of density function of $\operatorname{OLLBuX}-\mathrm{N}(\alpha, \theta, \mu, \sigma)$. 


\subsection{The OLLBuX-Weibull Family (OLLBuX-W)}

By taking $G(x ; \xi)$ and $g(x ; \xi)$ in (2) as the cdf and pdf of the Weibull distribution with $\operatorname{cdf} G(x ; \lambda, k)=1-e^{-\left(\frac{x}{\lambda}\right)^{k}}$ and with pdf $g(x ; \lambda, k)=$ $\frac{k}{\lambda}\left(\frac{x}{\lambda}\right)^{k-1} e^{-\left(\frac{x}{\lambda}\right)^{k}}$, where $k>0$ is a shape parameter, $\lambda>0$ is a scale parameter, and $\xi=(\lambda, k)^{T}$, the OLLBuX-W density if we suppose that $z(x)=1-\exp \left[-\left(e^{\left(\frac{x}{\lambda}\right)^{k}}-1\right)^{2}\right]$ follows as $(x>0)$

$$
f(x ; \alpha, \theta, \lambda, k)=\frac{2 \alpha \theta \frac{k}{\lambda}\left(\frac{x}{\lambda}\right)^{k-1} e^{-\left(\frac{x}{\lambda}\right)^{k}}\left(1-e^{-\left(\frac{x}{\lambda}\right)^{k}}\right)(1-z(x)) z(x)^{\alpha \theta-1}\left(1-z(x)^{\theta}\right)^{\alpha-1}}{e^{-3\left(\frac{x}{\lambda}\right)^{k}}\left\{z(x)^{\alpha \theta}+\left[1-z(x)^{\theta}\right]^{\alpha}\right\}^{2}} .
$$

In Figure 2 some density and hazard functions for OLLBuX-W have been drown.

\section{LINEAR COMBINATIONS}

Using generalized binomial expansion, for any $z \in(0,1)$ and $\alpha>0$, we can write as follows:

$$
z^{\alpha}=\sum_{k=0}^{\infty} s_{k}(\alpha) z^{k}
$$

where

$$
s_{k}(\alpha)=\sum_{i=k}^{\infty}(-1)^{i+k}\left(\begin{array}{c}
\alpha \\
i
\end{array}\right)\left(\begin{array}{c}
i \\
k
\end{array}\right)
$$

Then we can write as the following:

$$
\left\{1-\exp \left[-\left(\frac{G(x)}{\bar{G}(x)}\right)^{2}\right]\right\}^{\alpha \theta}=\sum_{k=0}^{\infty} a_{k}\left\{1-\exp \left[-\left(\frac{G(x)}{\bar{G}(x)}\right)^{2}\right]\right\}^{\theta k}
$$
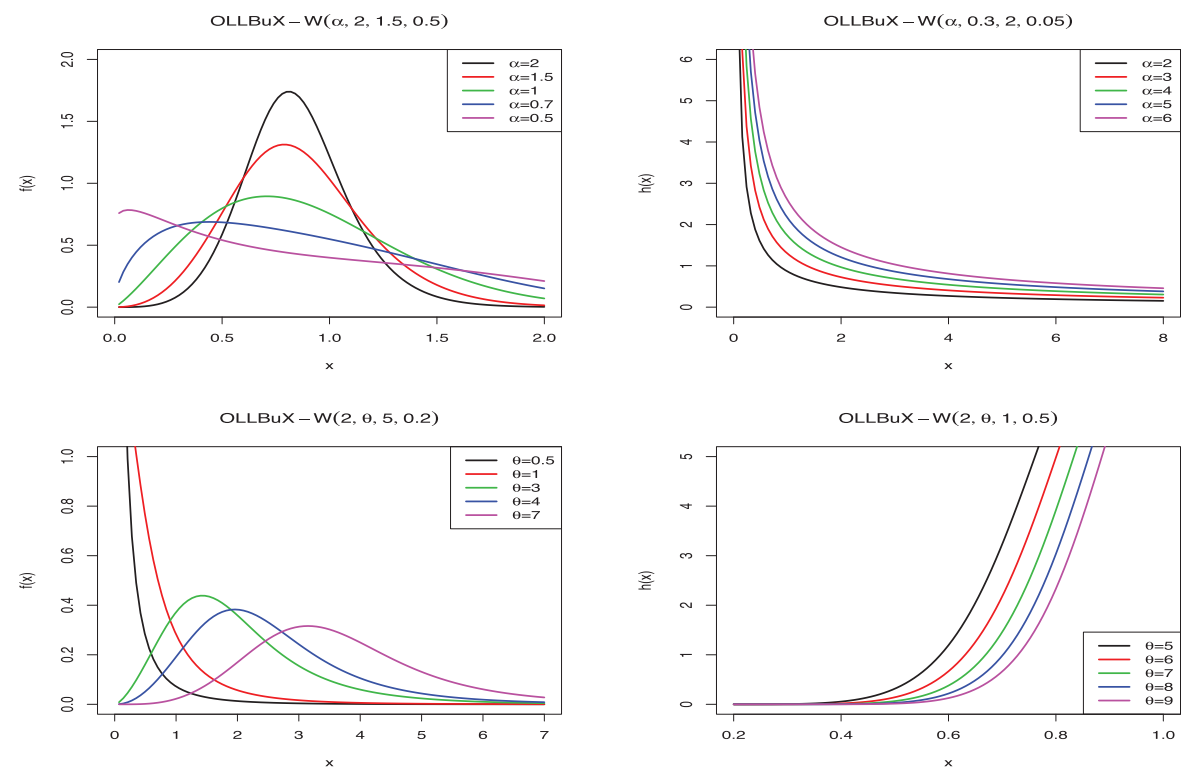

Figure 2 The sample curves of density and hazard function of $\operatorname{OLLBuX}-\mathrm{W}(\alpha, \theta, \lambda, k)$. 
where $a_{k}=s_{k}(\alpha)$ and

$$
\left\{1-\exp \left[-\left(\frac{G(x)}{\bar{G}(x)}\right)^{2}\right]\right\}^{\alpha \theta}+\left\{1-\left\{1-\exp \left[-\left(\frac{G(x)}{\bar{G}(x)}\right)^{2}\right]\right\}^{\theta}\right\}^{\alpha}=\sum_{k=0}^{\infty} b_{k}\left\{1-\exp \left[-\left(\frac{G(x)}{\bar{G}(x)}\right)^{2}\right]\right\}^{\theta k}
$$

where $b_{k}=a_{k}+(-1)^{k}\left(\begin{array}{l}\alpha \\ k\end{array}\right)$. So we can write as follows:

$$
F(x)=\frac{\sum_{k=0}^{\infty} a_{k}\left\{1-\exp \left[-\left(\frac{G(x)}{\bar{G}(x)}\right)^{2}\right]\right\}^{\theta k}}{\sum_{k=0}^{\infty} b_{k}\left\{1-\exp \left[-\left(\frac{G(x)}{\bar{G}(x)}\right)^{2}\right]\right\}^{\theta k}}=\sum_{k=0}^{\infty} c_{k}\left\{1-\exp \left[-\left(\frac{G(x)}{\bar{G}(x)}\right)^{2}\right]\right\}^{\theta k},
$$

where $c_{0}=\frac{a_{0}}{b_{0}}$ and for $k \geq 1$. Also we have as follows:

$$
\begin{aligned}
c_{k}=\frac{1}{b_{0}}\left[a_{k}-\frac{1}{b_{0}} \sum_{r=1}^{k} b_{r} c_{k-r}\right] \\
f(x)=\sum_{k=0}^{\infty} \frac{2 \theta k c_{k} g(x) G(x) \exp \left[-\left(\frac{G(x)}{\bar{G}(x)}\right)^{2}\right]}{\bar{G}(x)^{2}\left(1-\exp \left\{-\left[\frac{G(x)}{\bar{G}(x)}\right]^{3}\right\}\right)^{1-\theta k}} \\
=\sum_{k, j=0}^{\infty} \frac{2 \theta(-1)^{j} k c_{k} \bar{G}(x)^{-3}}{\exp \left\{(j+1)\left[\frac{G(x)}{\bar{G}(x)}\right]^{2}\right\}}\left(\begin{array}{c}
\theta k-1 \\
j
\end{array}\right) g(x) G(x) \\
=\sum_{k, j, l=0}^{\infty} \frac{2 \theta(-1)^{j+l}(j+1)^{l} k c_{k}}{l ! \bar{G}(x)^{3+2 l}}\left(\begin{array}{c}
\theta k-1 \\
j
\end{array}\right) g(x) G(x)^{2 l+1} \\
=\sum_{k, j, l, r=0}^{\infty} \frac{2 \theta(-1)^{j+l+r}(j+1)^{l} k c_{k}}{l !}\left(\begin{array}{c}
\theta k-1 \\
j
\end{array}\right)\left(\begin{array}{c}
-2 l-3 \\
r
\end{array}\right) g(x) G(x)^{2 l+r+1} \\
=\sum_{k, j, l, r=0}^{\infty} w_{k, j, l, r} h_{2 l+r+2}(x),
\end{aligned}
$$

where

$$
w_{k, j, l, r}=\frac{2 \theta(-1)^{j+l+r}(j+1)^{l} k c_{k}}{l !(2 l+r+2)}\left(\begin{array}{c}
\theta k-1 \\
j
\end{array}\right)\left(\begin{array}{c}
-2 l-3 \\
r
\end{array}\right) .
$$

Finally we can write as follows:

$$
F(x)=\sum_{k, j, l, r=0}^{\infty} w_{k, j, l, r} H_{2 \nmid+r+2}(x)
$$

where $H_{a}(x)=G(x)^{a}, h_{a}(x)=a g(x) G(x)^{a-1}$ denote the cdf and pdf of exp-G with power parameter $a$. 


\section{STATISTICAL PROPERTIES}

In this section, we obtain some statistical properties such as: asymptotics, moments, moment-generating function, incomplete moments. Established algebraic expansions to determine some structural properties of the OLLBuX family can be more efficient than computing those directly by numerical integration of its density function.

\subsection{Limit Behaviors}

Proposition 4.1.1. Let $a=\inf \{x \mid F(x)>0\}$ the asymptotics of $F(x), f(x)$, and $h(x)$, as $x \rightarrow a$ are given by the following:

$$
\begin{array}{lll}
F(x) \sim G(x)^{2 \alpha \theta} & \text { as } & \mathrm{x} \rightarrow \mathrm{a}, \\
f(x) \sim 2 \alpha \theta g(x) G(x)^{2 \alpha \theta-1} & \text { as } & \mathrm{x} \rightarrow \mathrm{a}, \\
h(x) \sim 2 \alpha \theta g(x) G(x)^{2 \alpha \theta-1} & \text { as } & \mathrm{x} \rightarrow \mathrm{a} .
\end{array}
$$

Proposition 4.1.2. The asymptotics of equations $F(x), f(x)$, and $h(x)$, as $x \rightarrow \infty a$ are given by the following:

$$
\begin{aligned}
& 1-F(x) \sim \theta^{\alpha} \mathrm{e}^{\frac{-\alpha}{\overline{\mathrm{G}}(\mathrm{x})^{2}}} \quad \text { as } \quad \mathrm{x} \rightarrow \infty \\
& f(x) \sim \frac{2 \alpha \theta^{\alpha} g(x) \mathrm{e}^{\frac{-\alpha}{\overline{\mathrm{G}}(x)^{2}}}}{\bar{G}(x)^{3}} \quad \text { as } \quad \mathrm{x} \rightarrow \infty, \\
& h(x) \sim \frac{2 \alpha \theta^{\alpha} g(x)}{\bar{G}(x)^{3}} \quad \text { as } \quad \mathrm{x} \rightarrow \infty .
\end{aligned}
$$

We can evaluate the effect of parameters on tails of distribution using these equations.

\subsection{Moments}

Henceforth, let $Y_{\gamma}$ be the exp-G random variable with positive power parameter $\gamma$, i.e., having density $h_{\gamma}(x)$. The $n$th ordinary moment of $X$ say $\mu_{r}^{\prime}=E\left(X^{r}\right)$, is determined from (6) as follows:

$$
\mu_{n}^{\prime}=\sum_{k, j, l, r=0}^{\infty} w_{k, j, l, r} E\left(Y_{2 l+r+2}^{n}\right),
$$

where

$$
E\left(Y_{\gamma}^{n}\right)=\gamma \int_{-\infty}^{\infty} x^{n} g(x) G(x)^{\gamma-1} d x
$$

can be evaluated at least numerically for most parent distributions.

\subsection{Moment-Generating Function}

The moment-generating function (mgf) $M(t)=E\left(e^{t X}\right)$ of $X$ follows from (6) as follows:

$$
M(t)=\sum_{k, j, l, r=0}^{\infty} w_{k, j, l, r} M_{2 l+r+2}(t),
$$

where $M_{\gamma}(t)$ is the mgf of $Y_{\gamma}$. Hence, $M(t)$ follows easily from the exp-G generating function.

\subsection{Incomplete Moments}

The $n$th incomplete moment of $X$, say $I_{n}(t)$, can be determined from (6) as follows:

$$
I_{n}(t)=\int_{-\infty}^{t} x^{n} f(x) d x=\sum_{k, j, l, r=0}^{\infty} w_{k, j, l, r} \int_{-\infty}^{t} x^{n} h_{2 l+r+2}(x) d x .
$$


The main applications of the first incomplete moment, $I_{1}(t)$, refer to the mean deviations and the Lorenz and Bonferroni curves. The mean deviations about the mean $\left[\delta_{1}=E\left(\left|X-\mu_{1}^{\prime}\right|\right)\right]$ and about the median $\left[\delta_{2}=E(|X-M|)\right]$ of $X$ are given by $\delta_{1}=2 \mu_{1}^{\prime} F\left(\mu_{1}^{\prime}\right)-2 I_{1}\left(\mu_{1}^{\prime}\right)$ and $\delta_{2}=\mu_{1}^{\prime}-2 I_{1}(M)$, respectively, where $\mu_{1}^{\prime}=E(X), M=F^{-1}\left(\frac{1}{2}\right)$ is the median of $X, F\left(\mu_{1}^{\prime}\right)$ is easily calculated from $(1)$ and $I_{1}(t)$ given by (7) for $n=1$. The quantity $M$ follows from the baseline quantile function (qf).

The Lorenz and Bonferroni curves are very useful in economics, reliability, demography, insurance and medicine. For a given probability $\pi>0$, they are defined by $L(\pi)=I_{1}(q) / \mu_{1}^{\prime}$ and $B(\pi)=L(\pi) / \pi$, respectively, where $q=F^{-1}(\pi)=Q(\pi)$ comes directly from the qf of $X$ given in Section 1. If $\pi$ is the proportion of units whose income is lower than or equal to $q, L(\pi)$ gives the proportion of total income volume accumulated by the set of units with an income lower than or equal to $q$, whereas the Bonferroni curve $B(\pi)$ gives the ratio between the mean income of this group and the mean income of the population.

\section{THE MAXIMUM LIKELIHOOD ESTIMATOR}

Several methods for parameter estimation can be used but the maximum likelihood is the most commonly employed. The MLE's enjoy desirable properties to construct confidence intervals for the model parameters. Large sample theory for these estimates delivers simple approximations that work well in finite samples. The commonly used goodness-of-fit statistics to compare fitted models are: the maximum $\log$-likelihood $\left(\hat{\ell}_{\max }\right)$, Akaike information criterion (AIC), consistent Akaike information criterion (CAIC), BIC, Anderson-Darling $\left(A^{*}\right)$ and Cramér-von Mises $\left(W^{*}\right)$, the last two described by Chen and Balakrishnan [15]. In general, the smaller the values of these statistics, the better the fit to the data. In this section we evaluate the maximum likelihood estimation (MLE) through the mean squared error and bias. In this section the maximum likelihood equations for estimating the parameters are calculated.

Let $x_{1}, \ldots, x_{n}$ be the observed values from the OLLBuX-N distribution with parameters $\alpha, \theta, \mu, \sigma$. Let $\Theta=(\alpha, \theta, \mu, \sigma)^{\top}$ be the $r \times 1$ parameter vector. According to the Equation (2), the log-likelihood function for the vector of parameters $\Theta=(\alpha, \theta, \mu, \sigma)^{\top}$, can be written as follows:

$$
\begin{aligned}
\ell(\Theta) & =\ln (\Theta)=\ln \prod_{i=1}^{n} f\left(x_{i}\right) \\
& =2 n \ln 2+n \ln \alpha+n \ln \theta+\sum_{i=1}^{n} \ln g\left(x_{i} ; \xi\right)+\sum_{i=1}^{n} \ln G\left(x_{i} ; \xi\right) \\
& -\sum_{i=1}^{n}\left(\frac{G\left(x_{i} ; \xi\right)}{\bar{G}\left(x_{i} ; \xi\right)}\right)^{2}+(\alpha \theta-1) \sum_{i=1}^{n} \ln \left\{1-\exp \left[-\left(\frac{G\left(x_{i} ; \xi\right)}{\bar{G}\left(x_{i} ; \xi\right)}\right)^{2}\right]\right\} \\
& +(\alpha-1) \sum_{i=1}^{n} \ln \left\{1-\left\{1-\exp \left[-\left(\frac{G\left(x_{i} ; \xi\right)}{\bar{G}\left(x_{i} ; \xi\right)}\right)^{2}\right]\right\}^{\theta}\right\}-3 \sum_{i=1}^{n} \ln \bar{G}\left(x_{i} ; \xi\right) \\
& -3 \alpha \theta \sum_{i=1}^{n} \ln \left\{1-\exp \left[-\left(\frac{G\left(x_{i} ; \xi\right)}{\bar{G}\left(x_{i} ; \xi\right)}\right)^{2}\right]\right\} \\
& -3 \alpha \sum_{i=1}^{n} \ln \left\{1-\left\{1-\exp \left[-\left(\frac{G\left(x_{i} ; \xi\right)}{\bar{G}\left(x_{i} ; \xi\right)}\right)^{2}\right]\right\}^{\theta}\right\}
\end{aligned}
$$

The log-likelihood function can be maximized either directly by solving the nonlinear likelihood equations obtained by differentiating (8). 
The components of the score function $U_{n}(\Theta)=\left(\frac{\partial \ell(\Theta)}{\partial \alpha}, \frac{\partial \ell(\Theta)}{\partial \theta}, \frac{\partial \ell(\Theta)}{\partial \xi}\right)^{\top}$ are the following:

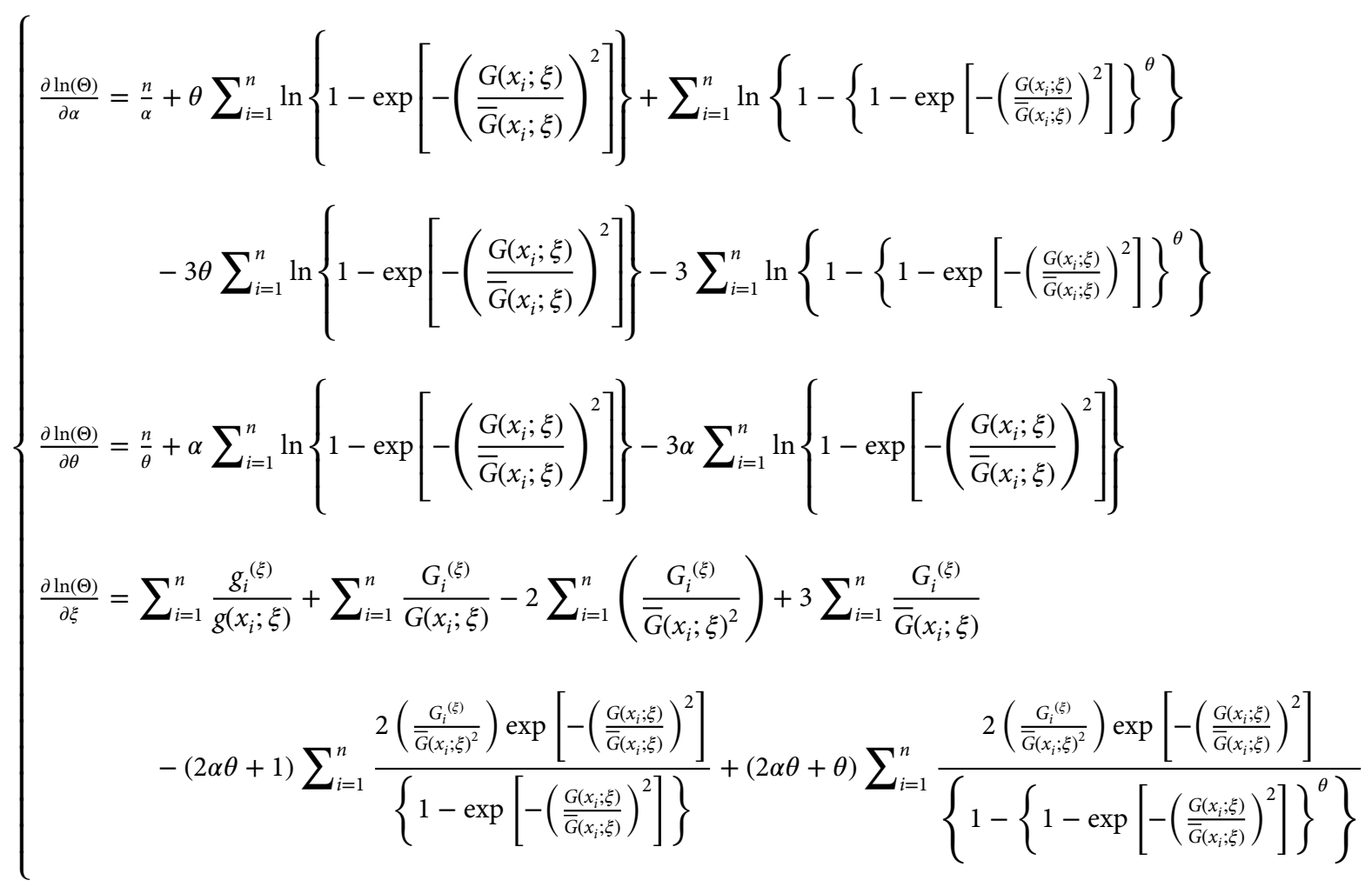

where $g_{i}^{(\xi)}=\frac{\partial g\left(x_{i} ; \xi\right)}{\partial \xi}$ and $G_{i}^{(\xi)}=\frac{\partial G\left(x_{i} ; \xi\right)}{\partial \xi}$

\section{SIMULATION STUDY}

\subsection{The Maximum Likelihood Estimator}

In this section, the Maximum likelihood estimators of parameters of purpose density function has been assessed by simulating: $(\alpha, \theta, \mu, \sigma)=$ $(0.9,2,1,2)$. The density function has been indicated in Figure 3.

To verify the check of the maximum likelihood estimator, the bias of MLE and the mean square error of MLE have been used. For example, as described in Section 5 , for $(\alpha, \theta, \mu, \sigma)=(0.9,2,1,2), r=1000$ times have been simulated samples of $n=10,11, \ldots, 60$ of

OLLBuX $-\mathrm{N}(0.9,2,1,2)$

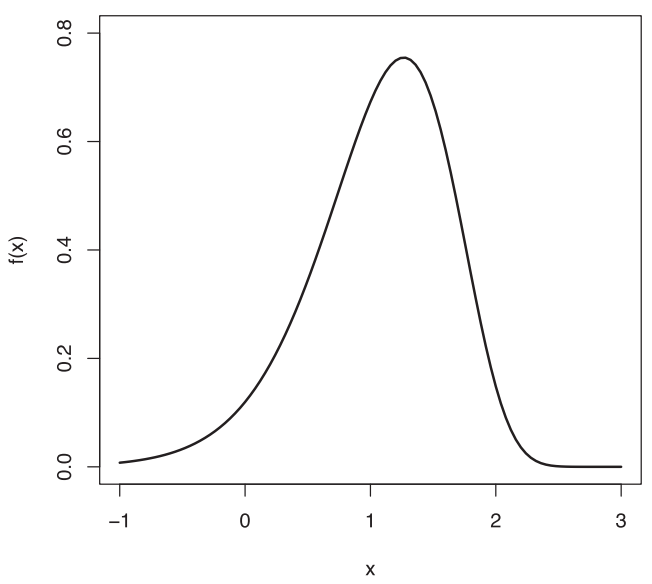

Figure 3 The density function for simulation study. 
OLLBuX-N $(0.9,2,1,2)$. To estimate the numerical value of the maximum likelihood, the optim function (in the stat package) and NelderMead method in R software has been used. If $\theta=(\alpha, \theta, \mu, \sigma)$, for any simulation by $n$ volume and $i=1,2, \ldots, r$, the maximum likelihood estimates are obtained as $\hat{\theta}_{i}=\left(\hat{\alpha}_{i}, \hat{\theta}_{i}, \hat{\mu}_{i}, \hat{\sigma}_{i}\right)$.

To investigate the performance of the MLE's for the OLLBuX-N distribution, we perform a simulation study:

(1) Generate $r$ samples of size $n$ from Equation (2).

(2) Compute the MLE's for the $r$ samples, say $(\hat{\alpha}, \hat{\theta}, \hat{\mu}, \hat{\sigma})$ for $i=1,2, \ldots, \mathrm{r}$.

(3) Compute the standard errors of the MLE's for $r$ samples, say $\left(s_{\hat{\alpha}}, s_{\hat{\theta}}, s_{\hat{\mu}}, s_{\hat{\sigma}}\right)$ for $i=1,2, \ldots, \mathrm{r}$.

(4) Compute the biases and mean squared errors given by

$$
\operatorname{Bias}_{\hat{\theta}}(n)=\frac{1}{r} \sum_{i=1}^{r}\left(\hat{\theta}_{i}-\theta_{i}\right)
$$

and

$$
\operatorname{MSE}_{\hat{\theta}}(n)=\frac{1}{r} \sum_{i=1}^{r}\left(\hat{\theta}_{i}-\theta_{i}\right)^{2},
$$

for $\theta=(\alpha, \theta, \mu, \sigma)$.

We repeat these steps for $r=1000$ and $n=10,11, \ldots n^{*}$ ( $n^{*}$ is different in each issue) with different values of $(\alpha, \theta, \mu, \sigma)$, so computing $\operatorname{Bias}_{\hat{\theta}}(n), \operatorname{MSE}_{\hat{\theta}}(n)$.

Figures 4 and 5 respectively reveals how the four biases, mean squared errors vary with respect to $n$. As expected, the Biases and MSEs of estimated parameters converges to zero while $n$ growing.

\subsection{The Other Estimation Methods}

There are several approaches to estimate the parameters of distributions that each of them has its characteristic features and benefits. In this subsection five of those methods are briefly introduced and will be numerically investigated in the simulation study Figure 3 . A useful summary of these methods can be seen in Dey et al. [16]. Here $\left\{t_{i} ; i=1,2, \ldots, n\right\}$ is the associated order statistics and $F$ is the distribution function of OLLBuX-N.
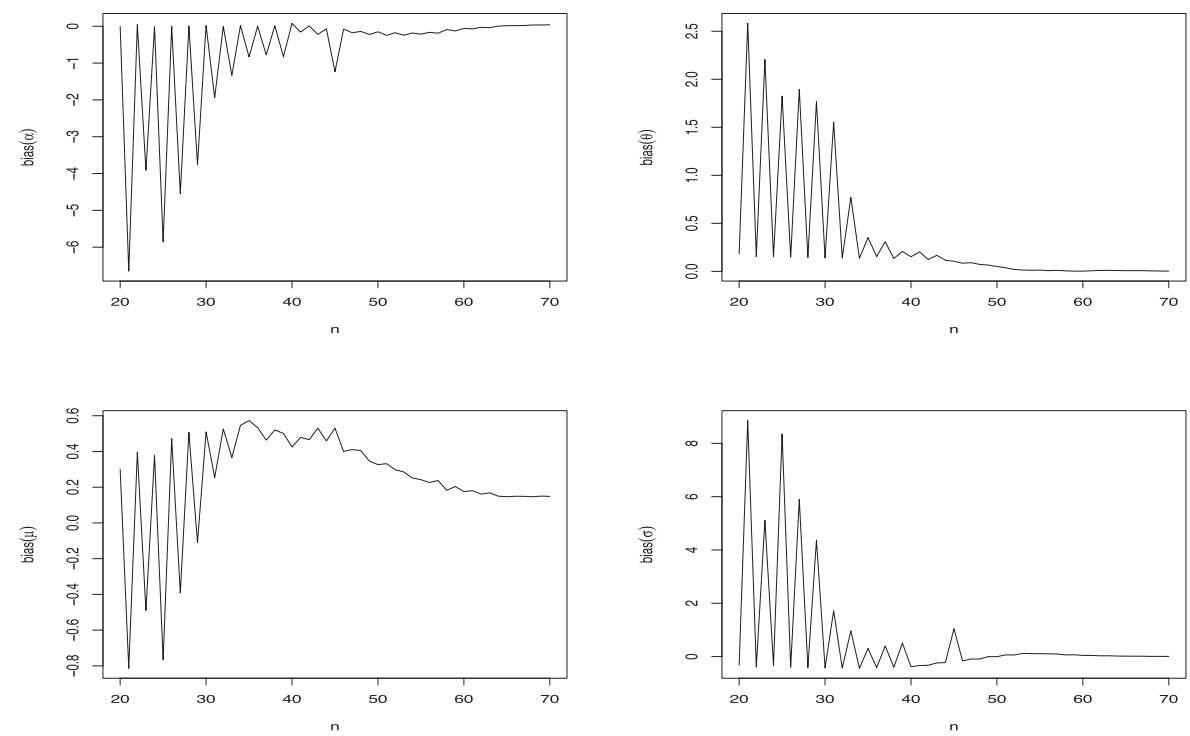

Figure 4 Bias of $\hat{\alpha}, \hat{\theta}, \hat{\mu}, \hat{\sigma}$ versus $n$ when $(\alpha, \theta, \mu, \sigma)=(0.9,2,1,2)$. 

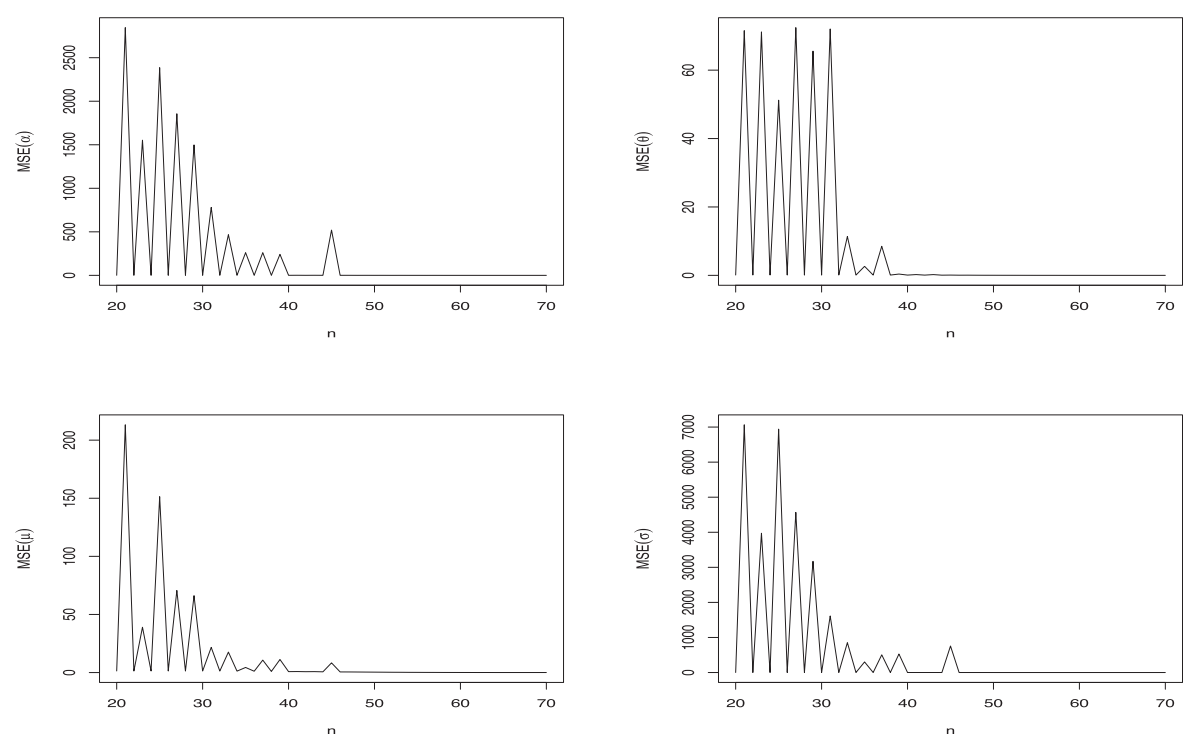

Figure 5 MSE of $\hat{\alpha}, \hat{\theta}, \hat{\mu}, \hat{\sigma}$ versus $n$ when $(\alpha, \theta, \mu, \sigma)=(0.9,2,1,2)$.

\subsubsection{Least squares and weighted least squares estimators}

The Least Squares (LSE) and weighted Least Squares Estimators (WLSE) are introduced by Swain et al. [17]. The LSE's and WLSE's are obtained by minimizing the following functions:

$$
S_{\mathrm{LSE}}(\alpha, \theta, \mu, \sigma)=\sum_{i=1}^{n}\left(F\left(t_{i} ; \alpha, \theta, \mu, \sigma\right)-\frac{i}{n+1}\right)^{2}
$$

and

$$
S_{\mathrm{WLSE}}(\alpha, \theta, \mu, \sigma)=\sum_{i=1}^{n} \frac{(n+1)^{2}(n+2)}{i(n-i+1)}\left(F\left(t_{i} ; \alpha, \theta, \mu, \sigma\right)-\frac{i}{n+1}\right)^{2} .
$$

\subsubsection{Cramér-von-Mises estimator}

Cramér-von-Mises Estimator (CME) is introduced by Choi and Bulgren [18]. The CMEs is obtained by minimizing the following function:

$$
S_{\mathrm{CME}}(\alpha, \theta, \mu, \sigma)=\frac{1}{12 n}+\sum_{i=1}^{n}\left(F\left(t_{i} ; \alpha, \theta, \mu, \sigma\right)-\frac{2 i-1}{2 n}\right)^{2} .
$$

\subsubsection{Anderson-Darling and right-tailed Anderson-Darling}

The Anderson Darling (ADE) and Right-Tailed Anderson Darling Estimators (RTADE) are introduced by Anderson and Darling [19]. The ADE's and RTADE's are obtained by minimizing the following functions:

$$
S_{\mathrm{ADE}}(\alpha, \theta, \mu, \sigma)=-n-\frac{1}{n} \sum_{i=1}^{n}(2 i-1)\left\{\log F\left(t_{i} ; \alpha, \theta, \mu, \sigma\right)+\log \bar{F}\left(t_{n+1-i} ; \alpha, \theta, \mu, \sigma\right)\right\}
$$

and

$$
S_{\mathrm{RTADE}}(\alpha, \theta, \mu, \sigma)=\frac{n}{2}-2 \sum_{i=1}^{n} F\left(t_{i} ; \alpha, \theta, \mu, \sigma\right)-\frac{1}{n} \sum_{i=1}^{n}(2 i-1) \log \bar{F}\left(t_{n+1-i} ; \alpha, \theta, \mu, \sigma\right),
$$

where $\bar{F}(\cdot)=1-F(\cdot)$.

In order to explore the estimators introduced above we consider the one model that have been used in this section, and investigate MSE of those estimators for different samples. For instance according to what has been mentioned above, for $(\alpha, \theta, \mu, \sigma)=(0.9,2,1,2)$. we have 
simulated $\mathrm{r}=1000$ times with sample size of the $n=50,55,60, \cdots 500$ and then the MSE formula that are mentioned in the subsection 6.1 are calculated for them. To obtain the value of the estimators, we have used the optima function and Nelder - Mead method in R. The result of the simulations of this subsection is shown in Figure 6. As it is clear from the MSE plot for two parameters with the increase in the volume of the sample all methods will approach to zero and this verifies the validity of the these estimation methods and numerical calculations for the distribution parameters OLLBuX-N.

\section{APPLICATIONS}

In this section, we submit two applications by fitting the OLLBuX-N model and some famous models. The AIC, Bayesian information criterion (BIC), Cramér-von Mises $\left(W^{*}\right)$, Anderson-Darling $\left(A^{*}\right)$, Kolmogorov-Smirnov (K.S) and the P-Value of K.S test, have been chosen for comparison of models for the first two examples.

The gamma-normal distribution (GaN), Alzaatreh et al. [20], The beta-normal distribution (BN), Eugene et al. [6], The McDonald normal distribution $(\mathrm{McN})$, Cordeiro et al. [21], Marshall-Olkin normal distribution (MON), Jose [22], The Kumaraswamy normal distribution $(\mathrm{KwN})$, Cordeiro and de Castro [23], The Zografos-Balakrishnan distribution (ZBN), Nadarajah et al. [24], and Log-normal distribution (LN), have been selected for comparison in the two examples. The parameters of models have been estimated by the MLE method.

\subsection{Phosphorus Concentration in Leaves Data Set}

This sub-section is related to study of the soil fertility in influence and the characterization of the biologic fixation of $\mathrm{N}_{2}$ for the Dimorphandra wilsonii rizz growth. For 128 plants, they made measures of the phosphorus concentration in the leaves (Fonseca and Franca [25]). The data, which have also been analyzed by Silva et al. [26]. The data set is the following: $0.22,0.17,0.11,0.10,0.15,0.06,0.05,0.07,0.12,0.09$, $0.23,0.25,0.23,0.24,0.20,0.08,0.11,0.12,0.10,0.06,0.20,0.17,0.20,0.11,0.16,0.09,0.10,0.12,0.12,0.10,0.09,0.17,0.19,0.21,0.18,0.26$, $0.19,0.17,0.18,0.20,0.24,0.19,0.21,0.22,0.17,0.08,0.08,0.06,0.09,0.22,0.23,0.22,0.19,0.27,0.16,0.28,0.11,0.10,0.20,0.12,0.15,0.08$, $0.12,0.09,0.14,0.07,0.09,0.05,0.06,0.11,0.16,0.20,0.25,0.16,0.13,0.11,0.11,0.11,0.08,0.22,0.11,0.13,0.12,0.15,0.12,0.11,0.11,0.15$, $0.10,0.15,0.17,0.14,0.12,0.18,0.14,0.18,0.13,0.12,0.14,0.09,0.10,0.13,0.09,0.11,0.11,0.14,0.07,0.07,0.19,0.17,0.18,0.16,0.19,0.15$, $0.07,0.09,0.17,0.10,0.08,0.15,0.21,0.16,0.08,0.10,0.06,0.08,0.12,0.13$.

In the Tables 1 and 2, a summary of the fitted information criteria and estimated MLE's for this data with different models have come, respectively. Models have been sorted from the lowest to the highest value of AIC. As you see, the OLLBuX-N is selected as the best model with more criteria. The histogram of the Phosphorus concentration in leaves data and the plots of fitted pdf are displayed in Figure 7.

\subsection{The Logarithm of the Egg Diameters of the Asteroids Data}

This sub-section is related to study of bimodal data set obtained from Emlet et al. [27] on the asteroid and echinoid egg size. The data consists of 88 asteroids species divided into three types; 35 planktotrophic larvae, 36 lecithotrophic larvae and 17 brooding larvae.
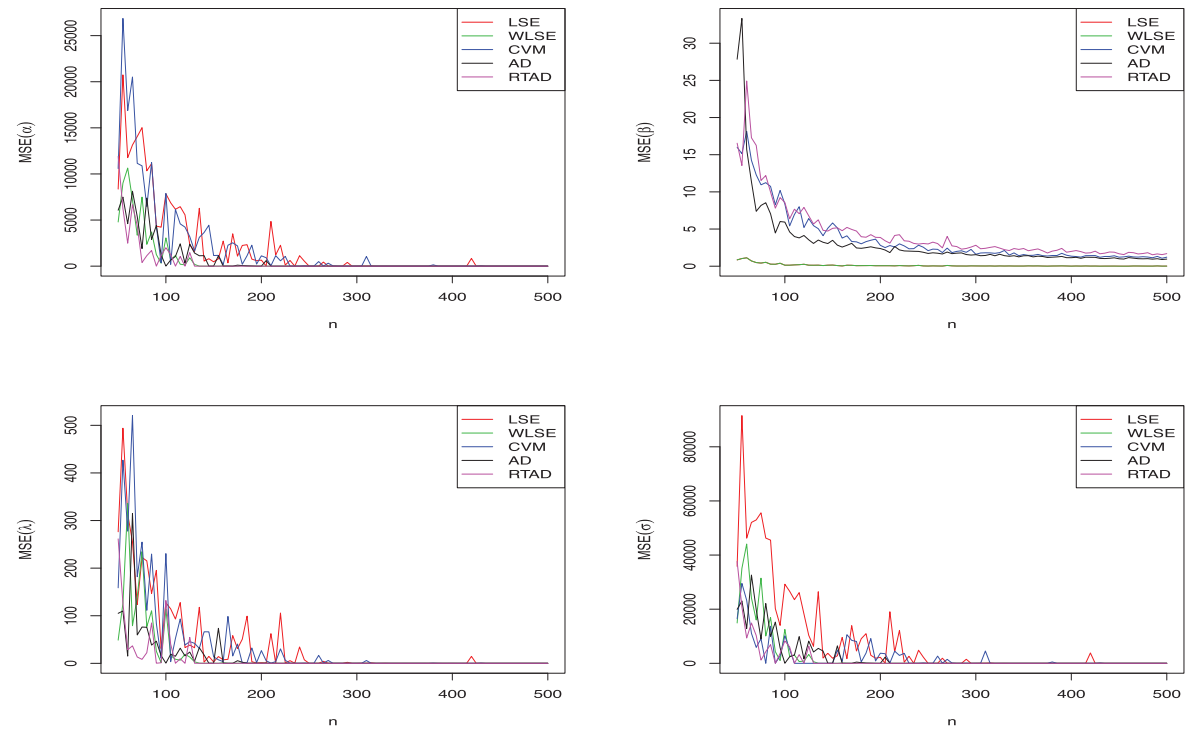

Figure 6 MSE of $\hat{\alpha}, \hat{\theta}, \hat{\mu}, \hat{\sigma}$ versus $n$ when $(\alpha, \theta, \mu, \sigma)=(0.9,2,1,2)$. 
Table 1 Information criteria for the phosphorus concentration in leaves data.

\begin{tabular}{lcccccc}
\hline Model & AIC & BIC & $W^{*}$ & $A^{*}$ & K.S & P-Value \\
\hline OLLBuX-N & -393.32 & -381.92 & 0.055 & 0.33 & 0.08 & 0.391 \\
KwN & -388.02 & -376.61 & 0.09 & 0.62 & 0.07 & 0.478 \\
BN & -387.96 & -376.55 & 0.09 & 0.62 & 0.077 & 0.441 \\
LN & -387.94 & -382.24 & 0.13 & 0.82 & 0.082 & 0.35 \\
McN & -386.07 & -371.81 & 0.17 & 0.96 & 0.30 & 0 \\
MON & -381.87 & -373.31 & 0.20 & 1.14 & 0.10 & 0.127 \\
ZBN & -379.47 & -370.91 & 0.41 & 2.75 & 0.946 & 0 \\
Normal & -379.04 & -373.34 & 0.27 & 1.56 & 0.13 & 0.021 \\
GaN & -371.97 & -360.57 & 0.38 & 2.56 & 1 & 0 \\
\hline
\end{tabular}

Table 2 Estimated MLEs and standard errors for the phosphorus concentration in leaves data.

\begin{tabular}{lcc}
\hline Model & MLE & Standard Errors \\
\hline OLLBuX-N $(\alpha, \theta, \mu, \sigma)$ & $(0.30,130.03,-0.03,0.33)$ & $(0.09,51.84,0.04,0.07)$ \\
$\operatorname{KwN}(\alpha, \theta, \mu, \sigma)$ & $(101.74,0.13,-0.02,0.04)$ & $(0.31,0.04,0.001,0.01)$ \\
$\operatorname{BN}(\alpha, \theta, \mu, \sigma)$ & $(73.21,0.13,-0.02,0.04)$ & $(49.68,0.03,0.01,0.003)$ \\
$\operatorname{LN}(\mu, \sigma)$ & $(-2.04,0.40)$ & $(0.04,0.03)$ \\
$\operatorname{McN}(\alpha, \theta, c, \mu, \sigma)$ & $(6.68,0.13,9.87,-0.02,0.04)$ & $(6.05,0.04,0.04,0.01,0.005)$ \\
$\operatorname{MON}(\alpha, \mu, \sigma)$ & $(0.28,0.18,0.06)$ & $(0.17,0.02,0.004)$ \\
$\operatorname{ZBN}(\alpha, \mu, \sigma)$ & $(4.82,-0.01,0.07)$ & $(6.43,0.18,0.01)$ \\
$\operatorname{Normal}(\mu, \sigma)$ & $(0.14,0.05)$ & $(0.004,0.003)$ \\
$\operatorname{GaN}(\alpha, \theta, \mu, \sigma)$ & $(21.95,0.01,0.57,0.45)$ & $(25.42,0.002,0.28,0.11)$ \\
\hline
\end{tabular}

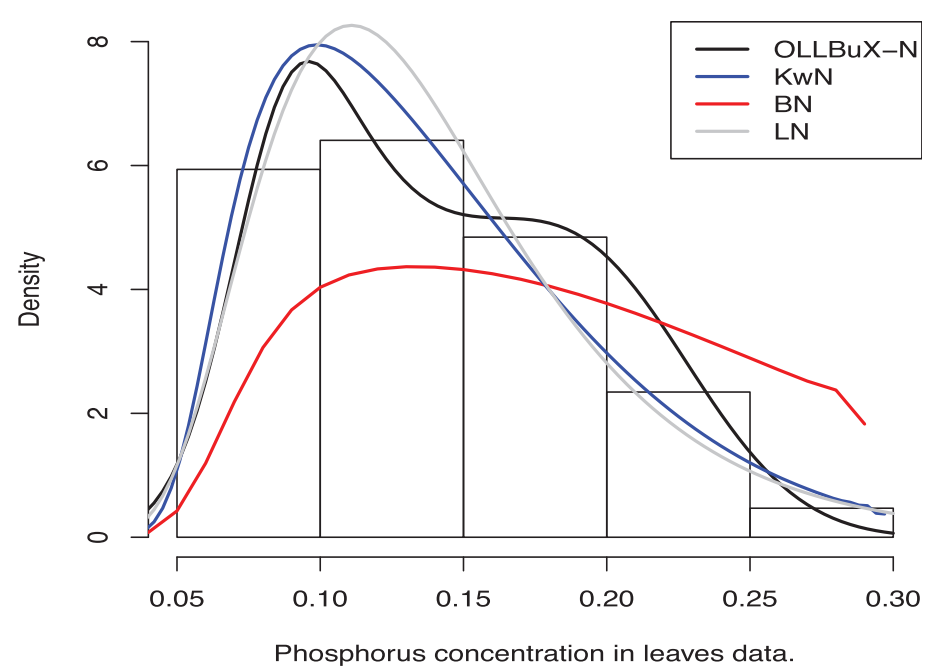

Figure 7 Histogram for Phosphorus concentration in leaves data set.

The logarithm of the egg diameters of the asteroids data has a bimodal shape.

Similar to the previous application example, we have Tables 3 and 4. As it is clear, the OLLBuX-N is selected as the best model with all criteria.

The histogram of the logarithm of the egg diameters of the asteroids data and the plots of fitted pdf are displayed in Figure 8.

\section{CONCLUSIONS}

A new class of distributions called the OLL Burr-X family with two extra positive parameters is introduced and studied. The new generator extends the OLL and Burr X distributions among several other well-known distributions. We provide some mathematical properties of the new family including moments and incomplete moments and generating function. Different methods have been used to estimate its parameters such as maximum likelihood, Least squares, weighted least squares, Cramer-von-Mises, Anderson-Darling and right-tailed Anderson-Darling methods. We assesses the performance of the maximum likelihood estimators in terms of biases and mean squared 
Table 3 Information criteria for the logarithm of the egg diameters of the asteroids data.

\begin{tabular}{lcccccc}
\hline Model & AIC & BIC & $W^{*}$ & $A^{*}$ & K.S & $\boldsymbol{P}$-Value \\
\hline OLLBuX-N & 227.12 & 237.03 & 0.16 & 0.94 & 0.14 & 0.191 \\
KwN & 244.38 & 254.29 & 0.44 & 2.40 & 0.15 & 0.039 \\
BN & 245.26 & 255.17 & 0.44 & 2.42 & 0.17 & 0.0151 \\
LN & 249.56 & 254.51 & 0.46 & 2.62 & 0.16 & 0.021 \\
GaN & 250.48 & 260.39 & 0.57 & 3.27 & 0.19 & 0.003 \\
Normal & 250.74 & 255.69 & 0.40 & 2.42 & 0.17 & 0.01 \\
ZBN & 252.002 & 259.43 & 0.82 & 4.71 & 0.827 & 0 \\
MON & 252.65 & 260.08 & 0.41 & 2.45 & 0.17 & 0.014 \\
McN & 256.22 & 268.61 & 0.42 & 2.48 & 0.91 & 0 \\
\hline
\end{tabular}

Table 4 Estimated MLEs and standard errors of the egg diameters of the asteroids data.

\begin{tabular}{ccc}
\hline Model & MLE & Standard Errors \\
\hline OLLBuX-N $(\alpha, \theta, \mu, \sigma)$ & $(0.18,103.73,3.79,4.12)$ & $(0.018,1.22,0.12,0.11)$ \\
$\operatorname{KwN}(\alpha, \theta, \mu, \sigma)$ & $(10.14,0.10,4.03,0.50)$ & $(0.14,0.01,0.05,0.002)$ \\
$\operatorname{BN}(\alpha, \theta, \mu, \sigma)$ & $(10.78,0.10,3.84,0.53)$ & $(3.60,0.01,0.002,0.002)$ \\
$\operatorname{LN}(\mu, \sigma)$ & $(1.79,0.16)$ & $(0.02,0.01)$ \\
$\operatorname{GaN}(\alpha, \theta, \mu, \sigma)$ & $(0.85,9.96,4.93,0.41)$ & $(0.12,1.10,0.01,0.02)$ \\
$\operatorname{Normal}(\mu, \sigma)$ & $(6.07,0.98)$ & $(0.10,0.07)$ \\
$\operatorname{ZBN}(\alpha, \mu, \sigma)$ & $(3.96,3.73,1.20)$ & $(7.10,4.60,0.25)$ \\
$\operatorname{MON}(\alpha, \mu, \sigma)$ & $(0.82,6.17,0.98)$ & $(0.54,0.36,0.07)$ \\
$\operatorname{McN}(\alpha, \theta, c, \mu, \sigma)$ & $(12.61,2.33,10.38,-1.81,3.57)$ & $(60.14,5.84,69.05,17.76,5.59)$ \\
\hline
\end{tabular}

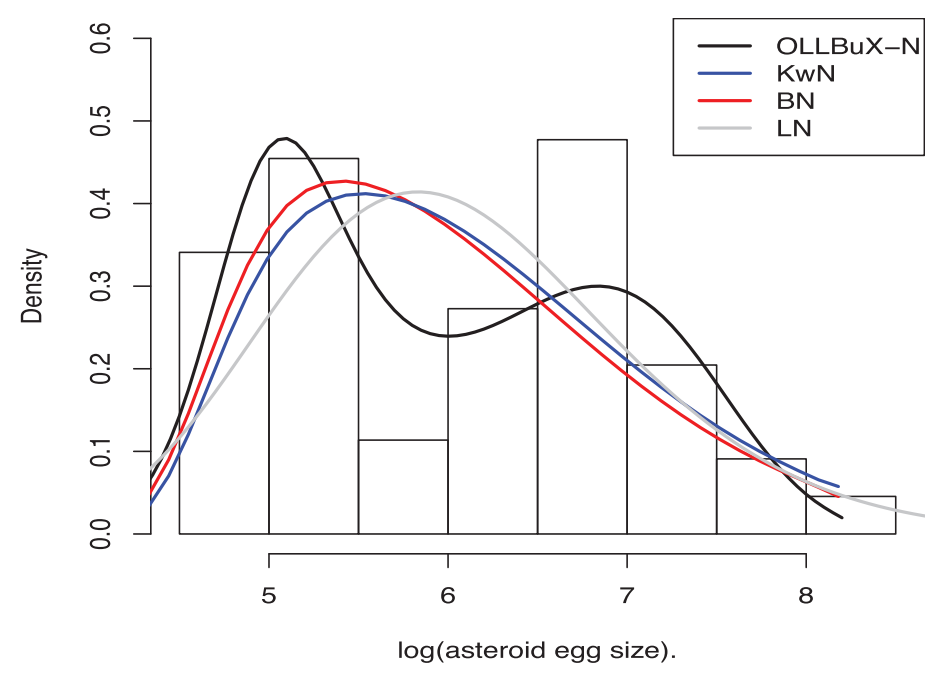

Figure 8 Histogram for the logarithm of the egg diameters of the asteroids data.

errors by means of a simulation study. Finally, the usefulness of the family is illustrated by means of two real data sets. The new models provide consistently better fits than other competitive models for these data sets. The new family is suitable for fitting different real data sets, the OLL Burr-X normal model is used for modeling bimodal and skewed data sets and can be sued as an alternative to the gamma-normal, beta-normal, McDonald-normal, Marshall-Olkin-normal, Kumaraswamy-normal, Zografos-Balakrishnan and log-normal distributions.

\section{CONFLICTS OF INTEREST}

The authors declare they have no conflicts of interest. 


\section{AUTHORS' CONTRIBUTIONS}

The authors have equally made contributions. All authors read and approved the final manuscript.

\section{ACKNOWLEDGMENTS}

The authors greatly appreciate both the Editor-in-Chief and the referees. This research is supported by Research Committee of Persian Gulf University.

\section{REFERENCES}

1. J.U. Gleaton, J.D. Lynch, J. Prob. Stat. Sci. 4 (2006), 51-64.

2. G.M. Cordeiro, M. Alizadeh, G. Ozel, B. Hosseini, E.M.M. Ortega, E. Altun, J. Stat. Comput. Simul. 87 (2017), $908-932$.

3. H. Haghbin, G. Ozel, M. Alizadeh, G.G. Hamedani, Commun. Stat. Theory Methods. 47 (2017), 9897-9920.

4. H.M. Yousof, A.Z. Afify, G.G. Hamedani, G. Aryal, J. Stat. Theory Appl. 16 (2017), 288-305.

5. A. Alzaatreh, C. Lee, F. Famoye, Metron. 71 (2013), 63-79.

6. N. Eugene, C. Lee, F. Famoye, Commun. Stat. Theory Methods. 31 (2002), 497-512.

7. M. Alizadeh, G.M. Cordeiro, A.D.C. Nascimento, M.D.S. Lima, E.M.M. Ortega, J. Stat. Comput. Simul. 87 (2017), $367-389$.

8. A.Z. Afify, G.M. Cordeiro, H.M. Yousof, A. Alzaatreh, Z.M. Nofal, J. Data Sci. 14 (2016), 245-270.

9. M. Ahsanullah, G.G. Hamedani, M. Shakil, B.M. Golam Kibria, F. George, J. Comput. Theor. Stat. 3 (2016), 63-73.

10. G.R. Aryal, H.M. Yousof, Stochast. Qual. Cont. 32 (2017), 7-23.

11. E. Brito, G.M. Cordeiro, H.M. Yousof, M. Alizadeh, G.O. Silva, J. Stat. Comput. Simul. 87 (2017), 3040-3058.

12. G.G. Hamedani, H.M. Yousof, M. Rasekhi, M. Alizadeh, S.M. Najibi, Pak. J. Stat. Oper. Res. 14 (2018), 39-55.

13. H. Karamikabir, M. Afshari, M. Alizadeh, G.G. Hamedani, Commun. Stat. Theory Methods. 50 (2021), 251-279.

14. H. Karamikabir, M. Afshari, H.M. Yousof, M. Alizadeh, G. Hamedani, J. Iran. Stat. Soc. 19 (2020), 121-161.

15. G. Chen, N. Balakrishnan, J. Qual. Technol. 27 (1995), 154-161.

16. S. Dey, J. Mazucheli, S. Nadarajah, J. Comput. Appl. Math. 37 (2017), 1-18.

17. J.J. Swain, S. Venkatraman, J.R. Wilson, J. Stat. Comput. Simul. 29 (1988), 271-297.

18. K. Choi, W. Bulgren, J. R. Stat. Soc. Ser. B. 30 (1968), 444-460.

19. T.W. Anderson, D.A. Darling, Ann. Math. Stat. 23 (1952), 193-212.

20. A. Alzaatreh, F. Famoye, C. Lee, Comput. Stat. Data Anal. 69 (2014), 67-80.

21. G.M. Cordeiro, R.J. Cintra, L.C. Rego, E.M.M. Ortega, Pak. J. Stat. Oper. Res. 8 (2012), 301-329.

22. K.K. Jose, in: International Statistical Institute: Proceeding of 58th World Statistical Congress (Session CPS005), Dublin, Ireland, 2011, pp. 3918-3923.

23. G.M. Cordeiro, M. de Castro, J. Stat. Comput. Simul. 81 (2011), 883-893.

24. S. Nadarajah, G.M. Cordeiro, E.M.M. Ortega, Commun. Stat. Theory Methos. 44 (2014), 186-215.

25. M.B. Fonseca, A influoencia da fertilidade do solo e caracterizacao da fixacao biologica de N2 para o crescimento de Dimorphandra wilsonii rizz, Master Thesis, Universidade Federal de Minas Gerais, Brazil, 2007.

26. R.B. Silva, M. Bourguignon, C.R.B. Dias, G.M. Cordeiro, Comput. Stat. Data Anal. 58 (2013), 352-367.

27. R.B. Emlet, L.R. McEdward, R.R. Strathmann, in: M. Jangouxand, J.M. Lawrence(Eds.), Echinoderm Studies 2, AA Balkema, Rotterdam, Netherlands, 1987, pp. 55-136. 